\title{
LONGITUDINAL SHEAR OF A BI-MATERIAL WITH FRICTIONAL SLIDING CONTACT IN THE INTERFACIAL CRACK
}

\author{
HEORHIY Sulym \\ Bialystok University of Technology, Bialystok, Poland; e-mail: h.sulym@pb.edu.pl \\ IAROSLAV PASTERNAK \\ Lutsk National Technical University, Lutsk, Ukraine; e-mail: pasternak@ukrpost.ua
}

Lyubov Piskozub, Yosyf Piskozub

Ukrainian Academy of Printing, Pidgolosko, L'viv, Ukraine; e-mail: piskozub@pancha.lviv.ua

\begin{abstract}
We construct an analytical solution to the anti-plane problem of an inhomogeneous bi-material medium with the interfacial crack considering sliding friction. The medium is exposed to an arbitrary normal and shear loading in the longitudinal direction. Using the jump function method, the problem is reduced to a solution to singular integral equations for the jumps of displacements and stresses in the areas with sliding friction. Explicit expressions for displacements, stress intensity factors and energy dissipation are obtained. Critical load values for determination of the onset of slippage are investigated. The effect of friction and loading parameters on the size of the slip zone, stress intensity factors and energy dissipation is numerically analyzed.
\end{abstract}

Keywords: friction, interfacial crack, stress intensity factor, longitudinal shear, jump functions

\section{Introduction}

Contact problems have received much attention in the literature as a result of their practical importance. The study of contact phenomena considering friction is one of the most pressing problems in engineering (Arhipenko and Kriviy, 2008; Goryacheva, 2001; Comninou, 1977; Ostryk and Ulitko, 2006; Sulym and Piskozub, 2004; Hills et al., 1993; Johnson, 1985; Kalker, 1977) and others. To a greater or lesser extent, but contact phenomena are always accompanied by friction at both macroscopic and microscopic levels. Mechanical, electrical, thermal, chemical processes and vibration that can simultaneously occur due to friction significantly affect degradation of materials, duration of their wave processes, reliability and durability of structural elements, etc. The effect of friction can be both harmful and helpful, when causing dissipation of the accumulated strain energy in the body and thus reduce stress.

However, the problem of contact interaction between adjacent surfaces of a crack has not received sufficient attention. Major achievements in this area include the study of theory of cracks at the interface of two media assuming the elimination of physically incorrect oscillating features singularity by a widely used model of local contact directly near the vicinity of the crack end (Comninou, 1977; Comninou et al., 1980; Comninou and Dundurs, 1980; Kundrat and Sulym, 2003; Cherepanov, 1966; Herrmann and Loboda, 1999; Kharun and Loboda, 2003). A wide class of problems on the effect of friction forces on the contact stresses between the half-planes was examined by Martynyak and Kryshtafovych (2000), Aravas and Sharma (1991), Weertman et al. (1983). Growth of cracks (in fact, the relative slip of materials) on the verge of a hard fibrous inclusion considering friction between the components was studied by Brussat and Westmann (1974). 
This paper proposes a novel technique for obtaining the analytical solution of the anti-plane problem (longitudinal shear) for a bi-material with a closed interfacial crack accounting for friction between surfaces. Therefore, all characteristics of the stress-strain state, such as displacements, stresses, energy dissipation, slip zone size, etc., are exactly calculated. Note that the frictional slippage is essentially an incremental process and, therefore, the solution to the contact problem depends on the load history. We assume that the magnitude and direction of the external force factors that generate longitudinal shear change quasi-statically (so slowly that it is not necessary to consider the inertial member) by a certain law, which may be different. Thus, no incremental formulation is necessary for solving this problem because of quasi-statically way of the initial step loading.

\section{Formulation of the problem}

Consider an infinite isotropic matrix consisting of two half-spaces with elastic constants $E_{k}, \nu_{k}$ $(k=1,2)$. The half-spaces are mutually pressed to the interface by external normal stresses $\sigma_{y y}(x)<0$. Here, Oxyz are the Cartesian coordinates and $x O z$ is the contact plane of the half-spaces.

We study the stress-strain state (SSS) of the body section by the plane $x O y$ perpendicular to the direction of its longitudinal shear. This section forms two half-planes $S_{k}(k=1,2)$, and the interface between them corresponds to the $x$-axis $L$. Under the action of the applied loads, the cracks may slip at intervals forming the line $L^{\prime}=\cup_{n=1}^{N} L_{n}^{\prime}=\cup_{n=1}^{N}\left[b_{n}^{-} ; b_{n}^{+}\right]$as indicated in Fig. 1. The normal stress in the body is generated by uniform compression at infinity $\sigma_{y y}^{\infty}<0$ and two balanced concentrated forces $P_{k}=\mp \mathrm{i} P$ at the points $z_{k} \in S_{k}$. The same traditional notation for the axis $z$ and a complex variable $z=x+\mathrm{i} y$ should not cause misunderstanding in the solution of the problem.

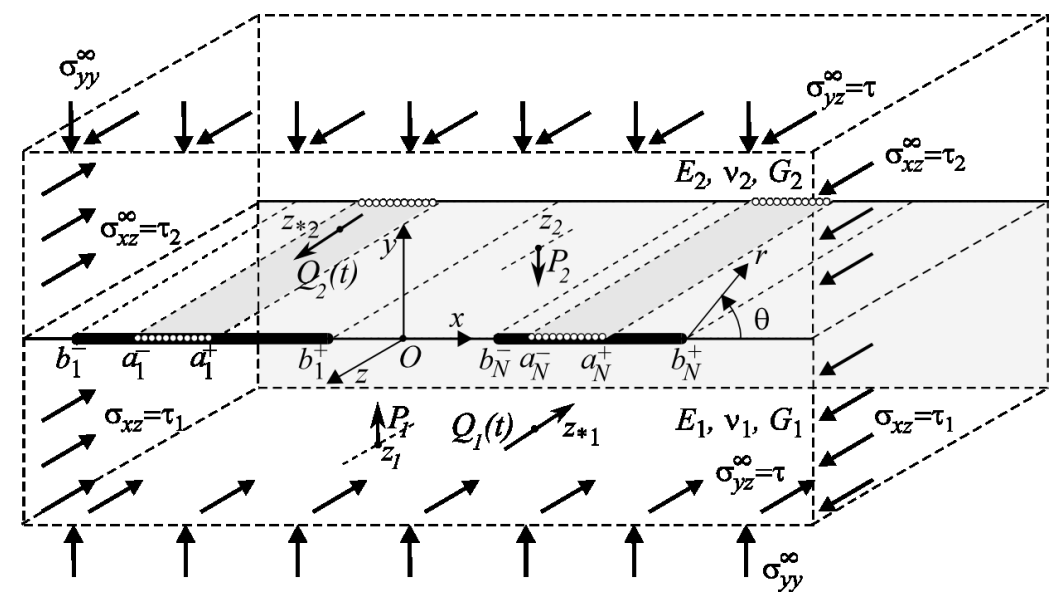

Fig. 1. Geometry and loading scheme of the problem

Suppose that the external loading increases or decreases monotonically and consists of a uniformly distributed at the infinity shear stress $\sigma_{y z}^{\infty}=\tau(t), \sigma_{x z}^{\infty}=\tau_{k}(t)$, concentrated forces with magnitude $Q_{k}(t)$, screw dislocations with Burger's vector $b_{k}(t)$ at the points $z_{* k} \in S_{k}$ $(k=1,2), t$ denotes the time as parameter. Note that the positive direction of the forces and Burgers vectors is chosen along the axis as opposed to implicitly accepted in some studies the opposite direction. According to Eq. (20.5) (Sulym, 2007), stresses at infinity must always satisfy the conditions $\tau_{2}(t) G_{1}=\tau_{1}(t) G_{2}$, which provides straightness of the matrix interface at infinity.

Contact between the half-spaces is assumed mechanically perfect omitting $L^{\prime}$ where it is more complicated. The contact in $L^{\prime}$ we assume mechanically perfect until the moment when 
relative sliding of the crack surfaces may start in some areas $\gamma_{n} \subset L_{n}^{\prime}$ (Johnson, 1985; Pasternak et al., 2010; Piskozub, 2014; Piskozub et al., 2014).

Thus, we formulate the problem of longitudinal shear with possible slip in the interfacial cracks under the action of an inhomogeneous distribution of compressive normal stresses and frictional forces on the surfaces of contact (line section $L$ ). These forces may cause in these apriority unknown slip zones heat emission, energy dissipation, wear, etc.

\section{The problem solution}

The presence of such slip zones in the cracks can be simulated by jumps of traction and displacement vectors at $L_{n}^{\prime}$ (Piskozub, 2014; Piskozub et al., 2014; Sulym, 2007; Sulym and Piskozub, 2004; Piskozub and Sulim, 2008)

$$
\llbracket \Xi \rrbracket \equiv \boldsymbol{\Xi}^{-}-\boldsymbol{\Xi}^{+}=\mathbf{f}^{n}(x, t) \quad x \in L_{n}^{\prime}
$$

where $\boldsymbol{\Xi}(z, t)=\left[\sigma_{y z}, \partial t / \partial x\right](z, t)$ is the state vector; $\mathbf{f}^{n}(x, t)=\left[f_{3}^{n}, f_{6}^{n}\right](x, t)$ is the jump vector. Hereinafter, the following notation is used: $\llbracket \varphi \rrbracket_{L}=\varphi(x,-0)-\varphi(x,+0),\langle\varphi\rangle_{L}=\varphi(x,-0)+$ $\varphi(x,+0)$; symbols "+" and "-" correspond to the threshold function on the top and bottom edges of the line $L$.

Based on Hooke's law, expression (3.1) results in

$$
\begin{aligned}
& \llbracket \sigma_{y z} \rrbracket_{L_{n}^{\prime}} \equiv \sigma_{y z}^{-}-\sigma_{y z}^{+}=f_{3}^{n}(x, t) \\
& \llbracket \frac{\partial w}{\partial x} \rrbracket_{L_{n}^{\prime}} \equiv \frac{\partial w^{-}}{\partial x}-\frac{\partial w^{+}}{\partial x}=\llbracket \frac{\sigma_{x z}}{G} \rrbracket_{L_{n}^{\prime}} \equiv \frac{\sigma_{x z}^{-}}{G_{1}}-\frac{\sigma_{x z}^{+}}{G_{2}}=f_{6}^{n}(x, t) \quad x \in \gamma_{n} \subset L_{n}^{\prime} \\
& f_{3}^{n}(x, t)=f_{6}^{n}(x, t)=0 \quad \text { if } \quad x \notin \gamma_{n}
\end{aligned}
$$

The boundary conditions at $L_{n}^{\prime}$ provide that the slipping starts at some zones $\gamma_{n}=\left[a_{n}^{-} ; a_{n}^{+}\right] \subset L_{n}^{\prime}$ when reaching the tangent stress $\sigma_{y z}$ of a certain critical value $\tau_{y z}^{\max }$, moreover, this threshold shear stress $\sigma_{y z}$ can not exceed $\tau_{y z}^{\max }$. Confining with classic Amonton's law of friction (Goryacheva, 2001; Hills et al., 1993; Johnson, 1985), consider the contact problem which states that everywhere in $\gamma_{n}$, the shear stresses (friction force) are equal

$$
\begin{array}{ll}
\sigma_{y z}^{ \pm}(x)=-\operatorname{sgn} \llbracket w \rrbracket \tau_{y z}^{\max }(x) & \tau_{y z}^{\max }(x)=-\alpha \sigma_{y y}(x) \\
\sigma_{y y}<0 & \left|w^{-}-w^{+}\right| \neq 0
\end{array}
$$

where $\alpha$ denotes the coefficient of sliding friction. Outside the domains $\gamma_{n}$, there is no slippage, and the magnitude of shear stresses does not exceed the maximum allowable level

$$
\left|\sigma_{y z}(x)\right| \leqslant \tau_{y z}^{\max }(x)
$$

The sign (direction of action) of shear stresses is chosen depending on the sign of the difference in the mutual displacement $\llbracket w \rrbracket$ at the source point of $L_{n}^{\prime}$.

The general case of normal pressing gives

$$
\tau_{y z}^{\max }(x)=4 \alpha\left(-\frac{\sigma_{y y}^{\infty}}{4}+\sum_{k=1}^{2} E_{j} \eta_{k} \operatorname{Re} \frac{N_{k}}{x-z_{k}}\right) \quad j=3-k
$$

where

$$
\begin{aligned}
N_{k} & =\frac{P_{k}}{e_{j k}}-\frac{\kappa_{k} \bar{P}_{k}-P_{k}}{e_{k j}} & \kappa_{k} & =4-3 \nu_{k} \\
\eta_{k} & =\frac{1}{8 \pi\left(1-\nu_{k}\right)} & e_{k j} & =2 \frac{G_{k}+\kappa_{k} G_{j}}{\left(1-\nu_{1}\right)\left(1-\nu_{2}\right)}
\end{aligned}
$$


Amonton's law of friction in classical form (3.3) provides, of course, simplification of the boundary conditions for the basic problem, but making use of more complex models of friction (Johnson, 1985; Sulym and Piskozub, 2004; Comninou et al., 1980), taking into account the wear, does not essentially complicate the process of solving

$$
\begin{aligned}
& \sigma_{y z}^{ \pm}(x, t)=\mp p_{k} f_{3}^{n}(x, t)-C g_{6}^{n}(x, t)+\sigma_{y z}^{0 \pm}(x, t) \\
& \sigma_{x z}^{ \pm}(x, t)=\mp C f_{6}^{n}(x, t)+p_{k} g_{3}^{n}(x, t)+\sigma_{x z}^{0 \pm}(x, t) \\
& g_{r}^{n}(z, t) \equiv \frac{1}{\pi} \int_{L_{n}^{\prime}} \frac{f_{r}^{n}(x, t)}{x-z} d x \quad p_{k}=\frac{G_{k}}{G_{1}+G_{2}} \quad C=G_{3-k} p_{k} \\
& \sigma_{y z}(z, t)+\mathrm{i} \sigma_{x z}(z, t)=\sigma_{y z}^{0}(z, t)+\mathrm{i} \sigma_{x z}^{0}(z, t)+\mathrm{i} p_{k} g_{3}^{n}(z, t)-C g_{6}^{n}(z, t)
\end{aligned}
$$

where $z \in S_{k}, r=3,6, k=1,2$.

The superscript "+" refers to $k=2$; "-" $-k=1$. The superscript "0" denotes the corresponding values in the solid body model without heterogeneity (cracks) under the same external loading (homogeneous solution). Hereinafter, the following notations (Piskozub et al., 2014; Sulym, 2007; Piskozub and Sulim, 2008) are used

$$
\begin{aligned}
& \sigma_{y z}^{0}(z, t)+\mathrm{i} \sigma_{x z}^{0}(z, t)=\tau(t)+\mathrm{i}\left[\tau_{k}(t)+D_{k}(z, t)+\left(p_{k}-p_{j}\right) \bar{D}_{k}(z, t)+2 p_{k} D_{j}(z, t)\right] \\
& D_{k}(z, t)=-\frac{Q_{k}(t)+\mathrm{i} G_{k} b_{k}(t)}{2 \pi\left(z-z_{* k}\right)} \quad z \in S_{k} \quad k=1,2 \quad j=3-k
\end{aligned}
$$

Using (3.6), (3.7) and boundary conditions (3.2), (3.3) at the domains $\gamma_{n}$, the problem reduces to a system of $2 N$ singular integral equations

$$
\begin{aligned}
& f_{3}^{n}(x, t)=0 \\
& g_{6}^{n}(x, t)=\frac{1}{2 C}\left(\left\langle\sigma_{y z}^{0}(x, t)\right\rangle+2 \operatorname{sgn} \llbracket w \rrbracket \tau_{y z}^{\max }(x)\right)
\end{aligned}
$$

whose solution is known (Sulym, 2007).

For a more detailed analysis of the problem solution, consider the partial case of the presence of a single $(N=1)$ crack $L_{1}^{\prime}=[-b ; b]$ with a symmetric zone of slippage $\gamma_{1}=[-a ; a](a \leqslant b)$ that can occur when symmetric load acts about the vertical axis.

The solution to (3.8) in this case, after calculating the corresponding integrals, have the exact form

$$
\begin{aligned}
f_{6}(x, t) & =\frac{1}{\pi C \sqrt{a^{2}-x^{2}}}\left\{\pi\left[\tau(t)-\alpha \operatorname{sgn} \llbracket w \rrbracket \sigma_{y y}^{\infty}\right] x\right. \\
& \left.+\sum_{k=1}^{2} p_{j} \operatorname{Im}\left[\left[Q_{k}(t)+\mathrm{i} G_{k} b_{k}(t)\right]\left(\frac{\sqrt{z_{* k}^{2}-a^{2}} x-z_{* k}}{+} 1\right)\right]\right\} \\
& +\frac{4 \alpha \operatorname{sgn} \llbracket w \rrbracket}{C \sqrt{a^{2}-x^{2}}} \sum_{k=1}^{2} E_{j} \eta_{k} \operatorname{Re}\left[N_{k}\left(\frac{\sqrt{z_{k}^{2}-a^{2}}}{x-z_{k}}+1\right)\right] \quad j=3-k \quad x \in[-a ; a]
\end{aligned}
$$

Here the function $\sqrt{z^{2}-a^{2}}$ is the branch which satisfies the condition $\sqrt{z^{2}-a^{2}} / z \rightarrow 1$ $z \rightarrow \infty$. Similar arguments are used to select the branches $\sqrt{z_{* k}^{2}-a^{2}}$ and $\sqrt{\bar{z}_{* k}^{2}-a^{2}}, k=1,2$. 
Thus, the expression for the jump displacement $\llbracket w \rrbracket$ is obtained by integration of Eq. (3.9)

$$
\begin{array}{ll}
\llbracket w \rrbracket & (x, t)=\int_{-a}^{x} f_{6(1)}(x, t) d x=-\frac{\tau(t)-\alpha \operatorname{sgn} \llbracket w \rrbracket \sigma_{y y}^{\infty}}{C} \sqrt{a^{2}-x^{2}} \\
& +\sum_{k=1}^{2} \frac{p_{j}}{\pi C} \operatorname{Im}\left\{\left[Q_{k}(t)+\mathrm{i} G_{k} b_{k}(t)\right] I_{2}\left(x, a, z_{* k}\right\}\right. \\
& +\frac{4 \alpha \operatorname{sgn} \llbracket w \rrbracket}{C} \sum_{k=1}^{2} E_{j} \eta_{k} \operatorname{Re}\left[N_{k} I_{2}\left(x, a, z_{k}\right)\right] \quad j=3-k \quad|x| \leqslant a
\end{array}
$$

and

$$
\begin{aligned}
& I_{2}(x, a, z)=\frac{\pi}{2}+\arcsin \frac{x}{a}+I(x, a, z) \\
& I(x, a, z) \equiv \sqrt{z^{2}-a^{2}} \int_{-a}^{x} \frac{d x}{\sqrt{a^{2}-x^{2}}(x-z)}=\mathrm{i} \operatorname{sgn}(\operatorname{Im} z) \ln \frac{a(z-x)}{a^{2}-x z-\mathrm{i} \sqrt{a^{2}-x^{2}} \sqrt{z^{2}-a^{2}}}
\end{aligned}
$$

Introducing mode 3 stress intensity factor (SIF) by expression $K_{3}=\lim _{r \rightarrow 0(\theta=0)} \sqrt{\pi r}\left(\sigma_{y z}\right.$ ), it is easy to obtain the analytical form for SIF in the case of the crack slip zone $[-a ; a] \subset L^{\prime}$

$$
\begin{aligned}
& K_{3}^{ \pm}(t)=\frac{1}{2 \sqrt{\pi a}} \int_{-a}^{a} \sqrt{\frac{a \pm x}{a \mp x}}\left[\left\langle\sigma_{y z}^{0}(x, t)\right\rangle+2 \operatorname{sgn} \llbracket w \rrbracket \tau_{y z}^{\max }(x)\right] d x=\sqrt{\pi a}\left[\tau(t)-\alpha \operatorname{sgn} \llbracket w \rrbracket \sigma_{y y}^{\infty}\right] \\
& +\frac{1}{\sqrt{\pi a}} \sum_{k=1}^{2} p_{j} \operatorname{Im}\left[\left[Q_{k}(t)+\mathrm{i} b_{k}(t) G_{k}\right]\left(\frac{\sqrt{z_{* k}^{2}-a^{2}}}{a \mp z_{* k}} \pm 1\right)\right] \\
& \quad+4 \alpha \operatorname{sgn} \llbracket w \rrbracket \sqrt{\frac{\pi}{a}} \sum_{k=1}^{2} E_{j} \eta_{k} \operatorname{Re}\left[N_{k}\left(\frac{\sqrt{z_{k}^{2}-a^{2}}}{a \mp z_{k}} \pm 1\right)\right] \quad j=3-k
\end{aligned}
$$

Now it is time to discuss the question about the apriority unknown size of the slip zone $a$. While increasing the magnitude of shear load from zero to maximum, there are three phases that are fundamentally different in view of slipping:

1) The combination of compressive and increasing shear load always fulfills condition (3.4). There is no slippage at all, and the cracks have no effect on SSS of the matrix.

2) The magnitude of shear load at the time point $t^{*}$ becomes sufficient for the occurrence of conditions (3.3) at least in a limited area $\gamma_{1}=[-a ; a](a \leqslant b)$. The magnitude of the load when the slippage first appears, will be called the first critical. To determine this critical value and the current size of the slip zone, one can put SIF (3.12) equal to zero (Cherepanov, 1966; Piskozub, 2014; Piskozub et al., 2014).

3) The magnitude of shear load at the time $t^{* *}$ makes the size of the slip zone coincide with the crack size $a=b$. Further growth will not lead to an increase in the slip zone, but singular stress occurs in the crack tip and, therefore, non-zero SIF exists. The magnitude of the load, when the size of the slip zone coincides with the crack size for the first time, will be called the second critical.

It is also possible that such a combination of the shear and compressing loading make conditions (3.3) arise instantly along the whole $L^{\prime}$ or along the $L^{\prime}$, except for the area $\gamma_{1}$. This shear load value will be called the threshold value. 
The analytical form of solution for all SSS components allows obtaining an analytical expression for the work of friction forces at the slip zone $\gamma_{1}$ for arbitrary loading

$$
\begin{gathered}
W^{d}(t)=-\int_{-a}^{a}\left|\tau_{y z}^{\max }(x) \llbracket w \rrbracket(x, t)\right| d x=-\frac{4 \alpha}{C} \int_{-a}^{a} \mid\left(-\frac{\sigma_{y y}^{\infty}}{4}+\sum_{k=1}^{2} E_{j} \eta_{k} \operatorname{Re} \frac{N_{k}}{x-z_{k}}\right) \\
\cdot\left(-\left[\tau(t)-\alpha \operatorname{sgn} \llbracket w \rrbracket \sigma_{y y}^{\infty}\right] \sqrt{a^{2}-x^{2}}+\sum_{k=1}^{2} \frac{p_{j}}{\pi} \operatorname{Im}\left\{\left[Q_{k}(t)+\mathrm{i} G_{k} b_{k}(t)\right] I_{2}\left(x, a, z_{* k}\right)\right\}\right. \\
\left.+4 \alpha \operatorname{sgn} \llbracket w \rrbracket \sum_{k=1}^{2} E_{j} \eta_{k} \operatorname{Re}\left[N_{k} I_{2}\left(x, a, z_{k}\right)\right]\right) \mid d x \quad j=3-k
\end{gathered}
$$

Suppose that the loading of the matrix is symmetric about the vertical axis: the focus points of the applied concentrated force are $z_{k}= \pm \mathrm{i} h \in S_{k}$ and $z_{* k}= \pm \mathrm{i} d(k=2,1)$. Thus, simplifying expressions (3.5), (3.9) and (3.10), one can write expressions for the most important components of SSS

$$
\begin{aligned}
& \llbracket w \rrbracket(x, t)=-\frac{\tau(t)-\alpha \operatorname{sgn} \llbracket w \rrbracket \sigma_{y y}^{\infty} \sqrt{a^{2}-x^{2}}}{C} \\
& +\sum_{k=1}^{2} \frac{p_{3-k}}{2 \pi C}\left[(-1)^{k} Q_{k}(t) \ln \frac{\sqrt{a^{2}+d^{2}}-\sqrt{a^{2}-x^{2}}}{\sqrt{a^{2}+d^{2}}+\sqrt{a^{2}-x^{2}}}+G_{k} b_{k}(t)\left(\frac{\pi}{2}+\arcsin \frac{x}{a}\right)\right] \\
& +\frac{2 \alpha \operatorname{sgn} \llbracket w \rrbracket}{C} P \gamma^{+} \ln \frac{\sqrt{a^{2}+h^{2}}-\sqrt{a^{2}-x^{2}}}{\sqrt{a^{2}+h^{2}}+\sqrt{a^{2}-x^{2}}} \quad|x| \leqslant a \\
& K_{3}^{ \pm}(t)=\sqrt{\pi a}\left[\tau(t)-\alpha \operatorname{sgn} \llbracket w \rrbracket \sigma_{y y}^{\infty}\right] \\
& +\frac{1}{\sqrt{\pi a}} \sum_{k=1}^{2} p_{3-k}\left(\frac{(-1)^{k} Q_{k}(t) a}{\sqrt{a^{2}+d^{2}}} \pm G_{k} b_{k}(t) \frac{\sqrt{a^{2}+d^{2}}-d}{\sqrt{a^{2}+d^{2}}}\right)+\sqrt{\pi a} \frac{4 \alpha \operatorname{sgn} \llbracket w \rrbracket P \gamma^{+}}{\sqrt{a^{2}+h^{2}}} \\
& W^{d}(t)=-\frac{\alpha}{C} \mid 4 \pi\left[\tau(t)-\alpha \operatorname{sgn} \llbracket w \rrbracket \sigma_{y y}^{\infty}\right]\left[\frac{a^{2} \sigma_{y y}^{\infty}}{8}-P \gamma^{+}\left(\sqrt{h^{2}+a^{2}}-h\right)\right] \\
& +\sigma_{y y}^{\infty}\left(\sqrt{d^{2}+a^{2}}-d\right) \sum_{k=1}^{2}(-1)^{k} p_{3-k} Q_{k}(t) \\
& -2 P \gamma^{+} \ln \frac{\sqrt{h^{2}+a^{2}} \sqrt{d^{2}+a^{2}}+h d+a^{2}}{\sqrt{h^{2}+a^{2}} \sqrt{d^{2}+a^{2}}+h d-a^{2}} \sum_{k=1}^{2}(-1)^{k} p_{3-k} Q_{k}(t) \\
& -8 \alpha \pi \operatorname{sgn} \llbracket w \rrbracket P^{2} \gamma^{+2} \ln \frac{h^{2}+a^{2}}{h^{2}}+4 \sigma_{y y}^{\infty} \alpha \pi \operatorname{sgn} \llbracket w \rrbracket P \gamma^{+}\left(\sqrt{h^{2}+a^{2}}-h\right) \mid
\end{aligned}
$$

Substituting $P_{k}=P=0$ in expressions (3.14), we obtain the known special case of the half-spaces compressed only by a uniform load $\sigma_{y y}^{\infty}<0$ at infinity (Piskozub, 2014; Piskozub et al., 2014). More interesting is the special case of normal pressing by the concentrated forces $P_{k}=\mp \mathrm{i} P$ only. Below we analyze expressions (3.14) for various special cases of shear loading. Hereinafter, $\tau^{*}, Q_{k}^{*}$ denotes the first critical load values; $\tau^{* *}, Q_{k}^{* *}-$ the second critical load values, $\tau^{* * *}, Q_{k}^{* * *}$ - threshold load values.

1) Suppose that there are only tangential shear stresses at infinity $\tau(t)>0$. Given that with an increasing load $\operatorname{sgn} \llbracket w \rrbracket=-1$, we get

$$
K_{3}(t)=\sqrt{\pi a}\left[\tau(t)-\alpha \operatorname{sgn} \llbracket w \rrbracket \sigma_{y y}^{\infty}\right]+\sqrt{\pi a} \frac{4 \alpha \operatorname{sgn} \llbracket w \rrbracket P \gamma^{+}}{\sqrt{a^{2}+h^{2}}}
$$

From expression (3.15), we obtain the condition for the start of slipping

$$
\sqrt{\pi a}\left[\tau(t)+\alpha \sigma_{y y}^{\infty}\right]-\sqrt{\pi a} \frac{4 \alpha P \gamma^{+}}{\sqrt{a^{2}+h^{2}}}=0
$$


Thus, the first critical value $\tau^{*}$ and size of the slip zone $a$ become

$$
\tau^{*}=\alpha\left(\frac{4 P \gamma^{+}}{h}-\sigma_{y y}^{\infty}\right) \quad a(t)=\sqrt{\frac{16 \alpha^{2} P^{2} \gamma^{+2}}{\left[\tau(t)+\alpha \sigma_{y y}^{\infty}\right]^{2}}-h^{2}}
$$

Substituting $a=b$ in (3.17) gives us the second critical value and the condition of non-zero SIF nascence

$$
\tau^{* *}=\alpha\left(\frac{4 P \gamma^{+}}{\sqrt{b^{2}+h^{2}}}-\sigma_{y y}^{\infty}\right)
$$

Analysis of Eqs. (3.17) and (3.18) shows however that $\tau^{*}>\tau(t)>\tau^{* *}$, which is devoid of physical meaning. This fact is easily explained since in this case of loading $\tau_{y z}^{\max }(x)$ achieves its own maximum at the point $x=0 \in L_{1}^{\prime}$ and, therefore, the uniform growth of magnitude $\tau(t)$ will exceed the value $\tau_{y z}^{\max }(0)$ at the last time point having generated the pre-slippage at the remotest location. This case of loading leads to the following conclusion: geometry of the problem must be changed by introducing into consideration two slip zones.

2) There is only one concentrated force $Q_{2}(t)$ growing from zero to $Q_{\max }$ and acting at the point $z_{* 2}=\mathrm{i} d$ in the upper half-space. And given the fact that with the increasing load, $\operatorname{sgn} \llbracket w \rrbracket=-1$ using $(3.14)_{1,2}$, we obtain

$$
\begin{gathered}
\llbracket w \rrbracket(x, t)=-\frac{\alpha \sigma_{y y}^{\infty}}{C} \sqrt{a^{2}-x^{2}}+\frac{p_{1} Q_{2}(t)}{\pi C} \ln \frac{\sqrt{a^{2}+d^{2}}-\sqrt{a^{2}-x^{2}}}{\sqrt{a^{2}+d^{2}}+\sqrt{a^{2}-x^{2}}} \\
-\frac{\alpha P \gamma^{+}}{2 \pi C} \ln \frac{\sqrt{a^{2}+h^{2}}-\sqrt{a^{2}-x^{2}}}{\sqrt{a^{2}+h^{2}}+\sqrt{a^{2}-x^{2}}} \quad|x| \leqslant a
\end{gathered}
$$

and

$$
K_{3}(t)=\sqrt{\pi a} \alpha \sigma_{y y}^{\infty}+\sqrt{\frac{a}{\pi}} \frac{p_{1} Q_{2}(t)}{\sqrt{a^{2}+d^{2}}}-\sqrt{\pi a} \frac{4 \alpha P \gamma^{+}}{\sqrt{a^{2}+h^{2}}}
$$

By equating SIF (3.20) to zero, we obtain the first critical value

$$
Q_{2}^{*}=\frac{\pi \alpha d}{p_{1}}\left(\frac{4 \gamma^{+} P}{h}-\sigma_{y y}^{\infty}\right)
$$

and the condition when slippage appears at the first time. The slip zone size is determined from the equation

$$
\alpha \sigma_{y y}^{\infty}+\frac{p_{1} Q_{2}(t)}{\pi \sqrt{a^{2}+d^{2}}}-\frac{4 \alpha P \gamma^{+}}{\sqrt{a^{2}+h^{2}}}=0
$$

Without the component $\sigma_{y y}^{\infty}$, one can obtain the exact solution

$$
a(t)=\sqrt{\frac{h^{2} d^{2}\left[Q_{2}(t)^{2}-Q_{2}^{* 2}\right]}{h^{2} Q_{2}^{* 2}-d^{2} Q_{2}(t)^{2}}}
$$

It is clear that there is no slippage when $Q_{\max (1)}<Q_{2}^{*}$. Substituting $a=b$ in Eq (3.22) gives us the second critical value and the condition of non-zero SIF nascence

$$
Q_{2}^{* *}=\frac{\pi \alpha \sqrt{d^{2}+b^{2}}}{p_{1}}\left(\frac{4 \gamma^{+} P}{\sqrt{h^{2}+b^{2}}}-\sigma_{y y}^{\infty}\right)
$$

or when the component $\sigma_{y y}^{\infty}$ is absent

$$
Q_{2}^{* *}=\frac{4 \pi \alpha \gamma^{+} P}{p_{1}} \frac{\sqrt{d^{2}+b^{2}}}{\sqrt{h^{2}+b^{2}}}=Q_{2}^{*} \frac{h \sqrt{d^{2}+b^{2}}}{d \sqrt{h^{2}+b^{2}}}
$$


So, taking $Q_{\max } \geqslant Q_{2}^{* *}$ in general, we get the following scenario of changing the SSS:

- when $Q_{2}^{*} \leqslant Q_{2}(t) \leqslant Q_{2}^{* *}$, the jump of displacements $\llbracket w \rrbracket(x, t)$ and the slip zone size $a(t)$ are defined from Eqs. (3.19), (3.23), thus expression $(3.14)_{3}$ for calculating the energy dissipation takes form

$$
\begin{aligned}
& W^{d}(t)=-\frac{\alpha}{C} \mid 4 \pi \alpha \sigma_{y y}^{\infty}\left(\frac{a^{2} \sigma_{y y}^{\infty}}{8}-P \gamma^{+}\left(\sqrt{h^{2}+a^{2}}-h\right)\right) \\
& +\sigma_{y y}^{\infty}\left(\sqrt{d^{2}+a^{2}}-d\right) p_{1} Q_{2}(t)-2 P \gamma^{+} p_{1} Q_{2}(t) \ln \frac{(h+d)\left[4 \pi \alpha \gamma^{+} P-p_{1} Q_{2}(t)\right]}{(h-d)\left[4 \pi \alpha \gamma^{+} P+p_{1} Q_{2}(t)\right]} \\
& +8 \alpha \pi P^{2} \gamma^{+2} \ln \frac{16 \pi^{2} \alpha^{2} \gamma^{+2} P^{2}\left(h^{2}-d^{2}\right)}{h^{2}\left[16 \pi^{2} \alpha^{2} \gamma^{+2} P^{2}-p_{1}^{2} Q_{2}^{2}(t)\right]}-4 \sigma_{y y}^{\infty} \alpha \pi P \gamma^{+}\left(\sqrt{h^{2}+a^{2}}-h\right) \mid
\end{aligned}
$$

- when $Q_{2}^{* *} \leqslant Q_{2}(t) \leqslant Q_{\max }$, the size $a(t)$ in formulas (3.19), (3.20) and (3.26) should be replaced by $b$ instead of (3.23), then we have $a=b$ and (3.20) defines a non-zero SIF.

To determine the threshold load, it is enough to direct $a \rightarrow \infty$ in formula (3.25)

$$
Q_{2}^{* * *}=\frac{4 \pi \alpha \gamma^{+} P}{p_{1}}
$$

For a more detailed analytical analysis, we suppose that the component $\sigma_{y y}^{\infty}$ is absent. Having found that for the considered problem statement which provides a single sliding zone $\gamma_{1}=[-a ; a]$ $(a \leqslant b)$, the condition $h>d$ must consider the increasing load magnitude $Q_{2}(t) \geqslant Q_{2}^{*}$. Thus, noting the correlation $1 \leqslant\left(h \sqrt{d^{2}+b^{2}}\right) /\left(d \sqrt{h^{2}+b^{2}}\right) \leqslant h / d$, one can conclude that the size of the slip zone will coincide with the crack size before the time when the ratio $Q_{2}(t) / Q_{2}^{*}$ reaches the value $h / d$. Taking the value $h=d$, we obtain the threshold case of the third phase: $Q_{2}^{*}=Q_{2}^{* *}=Q_{2}^{* * *}$. The choice of parameter values $h<d$ requires changing the geometry of the considered problem to the case with the arising slip zone along the $L^{\prime}$, except for the area $\gamma_{1}=[-a ; a](a \leqslant b)$.

Similar reasoning for such cases of shear loading as that for the concentrated force acting at the point $z_{* 1}=-\mathrm{i} d$ in lower half-space, a pair of mutually opposite or collinear balanced concentrated forces acting in different semi-spaces and simultaneously increasing from zero to $Q_{\max }$ etc., provides similar to Eq (3.19)-(3.27) expressions for the components of SSS.

When the materials of the half-spaces are identical $\left(G_{1}=G_{2}=G\right)$, it is enough to assume in the above formulas $C=G / 2, p_{1}=p_{2}=1 / 2$. For smooth contact between the half-spaces one should put $\alpha=0$ in the above formulas. This immediately gives an instant increase of the slip size to the whole area $L^{\prime}$ for any asymmetric shear loading.

Note that the superposition of the obtained above solutions for different kinds of loading can not be used because of nonlinearity of the problem.

\section{Numerical analysis}

Using the above-mentioned approach, we determine the dependence of the slip zone size, shape of the displacement jump, energy dissipation and SIF on the basic parameters of SSS (distance and magnitude of the applied force, friction coefficient, ratio of elastic properties) in the most illustrative example of loading No. 2. To apply formulas (3.20)-(3.27), we introduce dimensionless values: size of the slip zone $a / b$, the coordinate and distance from the crack of the points of application of the shear and pressing forces respectively $x / b, d / b, h / b ; Q_{2}(t) / Q_{2}^{* * *}$ and $Q_{2}(t) / Q_{2}^{*}$ - the absolute and relative intensity of shear magnitude; $\llbracket w \rrbracket(x) C /\left(2 \alpha \gamma^{+} P\right), W^{d}(t) C /\left(8 \pi \gamma^{+2} P^{2}\right)$ and $K_{3} \sqrt{b} /\left(4 \sqrt{\pi} \alpha \gamma^{+} P\right)$ - the displacement jump, energy dissipation and SIF, respectively. 
The dependence of size $a / b$ on the relative magnitude of the applied force $Q_{2}(t) / Q_{2}^{* * *}$ is shown in Fig. 2a for different values $d / b, h / b$. It is noticeable that the growth rate of the size increases when $d / b$ approaches $h / b$. Growing distance from the crack position of the coordinate $h / b$ also leads to an increase in the rate of growth $a / b$.

Note that while $Q_{2}(t) / Q_{2}^{*} \leqslant 1$, there is no slippage. The slippage area increases monotonically and simultaneously with an increase in the load.
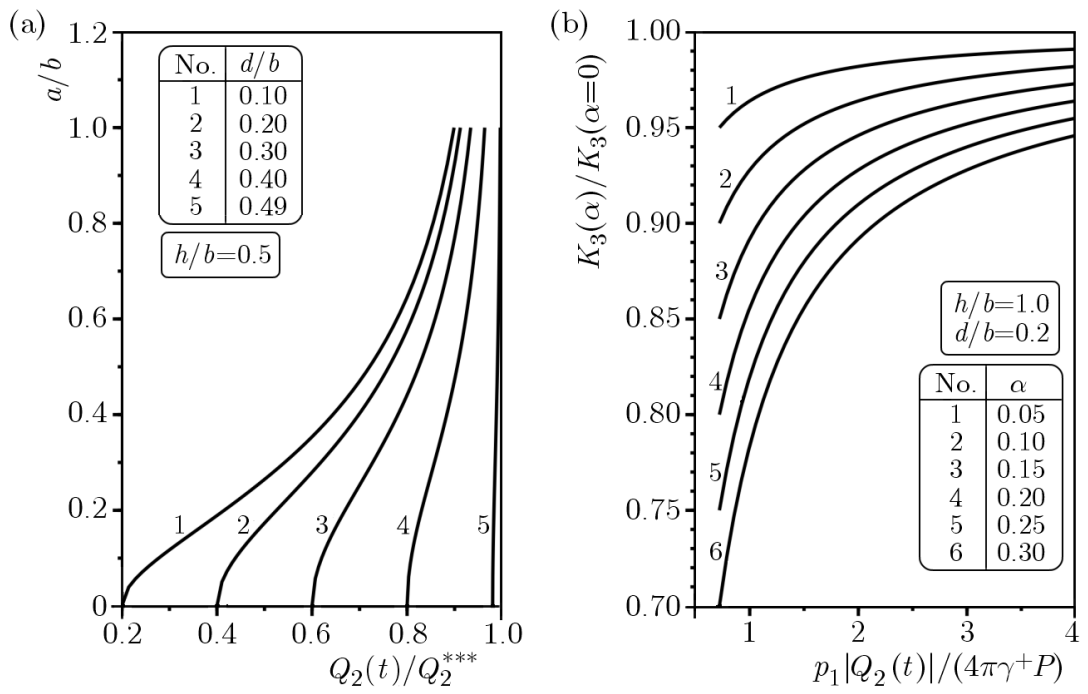

Fig. 2. (a) Dependence of the slip zone size on the coordinates of points of application of concentrated forces; (b) influence of friction in the slip zone on SIF

When the loading force magnitude excesses the second critical value (phase 3), then non-zero SIF in the vicinity of the crack tip appears. The calculated SIF is compared with the known SIF for an interfacial crack in the absence of friction, and it is shown in Fig. 2b. The presence of friction allows significant reduction of SIF (decline by 30\%).
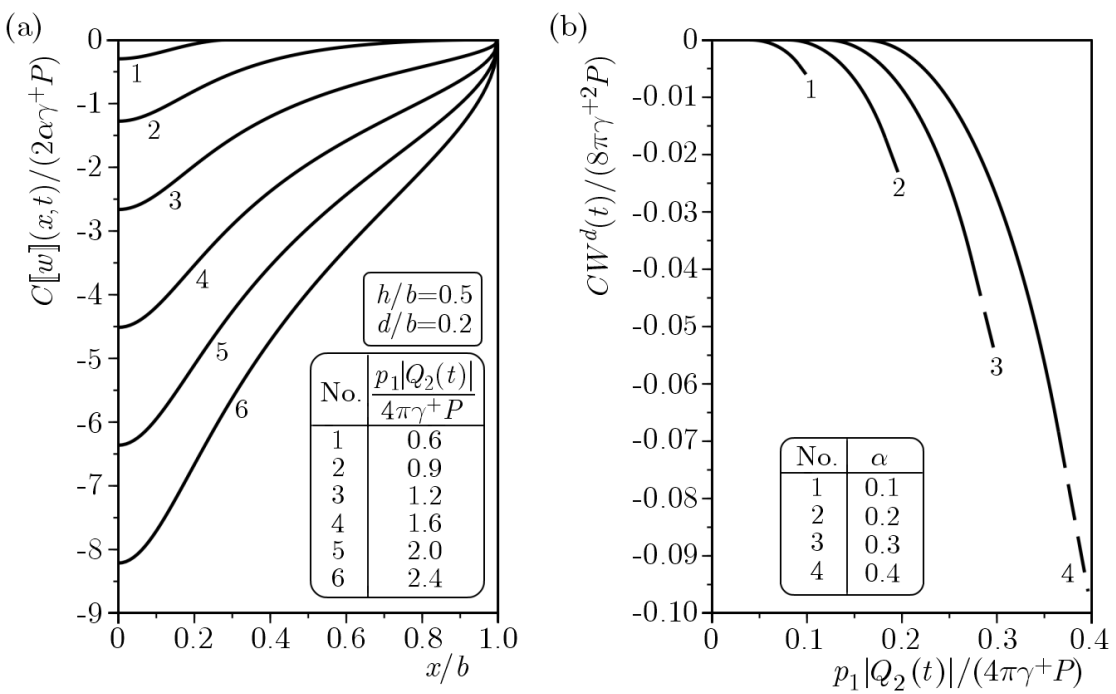

Fig. 3. (a) Shape of the displacement jump depending on the shear magnitude; (b) changes of the energy dissipation vs. load and coefficient of friction

Figure 3a depicts the influence of different settings on the shape of the displacement jump. It is noticeable that the highest sensitivity to the changing of the shape of the displacement jump is observed when placing the shear force application point closer to the crack. Approaching the 
points of application of normal forces lessens the effect on the shape of the displacement jump, but significantly changes its amplitude.

Figure $3 \mathrm{~b}$ indicates the energy dissipation during the second phase of loading by solid lines and by the dotted line - energy dissipation during the third phase of shear loading after reaching the maximum size of the slip zone. One observes the tendency of increasing the dissipation of energy when the coefficient of friction grows, or when approaching the point of force application to the crack position, or when the points of application of clamping forces are withdrawn.

\section{Conclusions}

We build an effective solution to the problem of a bi-material with a closed interfacial crack where sliding friction is possible. Different ways of loading of the solid body by arbitrary normal compression and monotonically increasing loading in the longitudinal direction is taken into account. This solution allows obtaining explicit expressions for displacements, stress intensity factors and energy dissipation. The dependence of the contact zone size on the loading parameters at different stages is analyzed. The critical load values for determination of the onset of slipping are investigated. Upon reaching the second critical value of the load when the slip zone size matches the size of the crack, the singular stresses in the vicinity of the ends of the crack and non-zero values of stress intensity factors appear.

We numerically analyze the effect of friction and loading parameters on the size of the slip zone, energy dissipation and stress intensity factors. It is discovered that the slip zone appears and grows fastest when the pressing normal stresses are minimal. The growth rate of the slip zone also promotes the increase of the distance of the application point of concentrated loading factors from it. The growth of the coefficient of friction significantly reduces the intensity of stresses at the vicinity of the ends of cracks at the third stage of loading. Energy dissipation for the examined cases of loading is calculated. The energy dissipation becomes more intense when the point of force application is closer to the sliding zone.

\section{References}

1. Aravas N., Sharma S.M., 1991, An elastoplastic analysis of the interface crack with contact zones, Journal of the Mechanics and Physics of Solids, 39, 311-344

2. Arhipenko K.M., Kriviy O.F., 2008, Interphase beam at different types of contact interaction of heterogeneous anisotropic plane (in Ukrainian), Mashynoznavstvo, 3, (129), 16-21

3. Brussat T.R., Westmann R.A., 1974, Interfacial slip around rigid fiber inclusion, Journal of Composite Materials, 8, 4, 364-377

4. Cherepanov G.P., 1966, On the development of cracks in compressed bodies (in Russian), Prikladnaya Matematika i Mehanika, 30, 1, 82-93

5. Comninou M., 1977, The interface crack, Journal of Applied Mechanics, 44, 631-636

6. Comninou M., Dundurs J., 1980, Effect of friction on the interface crack loaded in shear, Journal of Elasticity, 10, 2, 203-212

7. Comninou M., Schmueser D., Dundurs J., 1980, Frictional slip between a layer and a substrate caused by a normal load, International Journal of Engineering Science, 18, 131-137

8. Goryacheva I.G., 2001, Mechanics of Friction Interaction (in Russian), Moscow, Nauka

9. Herrmann K.P., Loboda V.V., 1999, On interface crack models with contact zones situated in an anisotropic bimaterial, Archive of Applied Mechanics, 69, 317-335 
10. Hills D.A., Nowell D., SACKfield A., 1993, Mechanics of Elastic Contact, ButterworthHeinemann, Oxford

11. Johnson K.L., 1985, Contact Mechanic, Cambridge etc., Cambridge University Press

12. Kalker J.J., 1977, A survey of the mechanics of contact between solid bodies, Zeitschrift für Angewandte Mathematik und Mechanik, 57, 5, T3-T17

13. Kharun I.V., Loboda V.V., 2003, A set of interface cracks with contact zones in combined tension-shear field, Acta Mechanica, 166, 43-56

14. Kundrat M.M., Sulym H.T., 2003, Prefracture zone in the composition of high-modulus elastic inclusion in the symmetric and antisymmetric load (in Ukrainian), Matematychni Problemy Mehaniky Neodnoridnyh Struktur, Lviv, 322-324

15. Martynyak R., Kryshtafovych A., 2000, Friction contact of two elastic half-planes with local recesses in boundary, Journal of Friction and Wear, 21, 4, 6-15

16. Ostryk V.I., Ulitko A.F., 2006, Wiener-Hopf Method in Contact Problems of Elasticity Theory (in Russian), Kiev, Naukova Dumka

17. Pasternak Ya.M., Sulym H.T., Piskozub L.G., 2010, Models of thin inclusion with the account of imperfect contact with the medium (in Russian), Proceedings of VI International Symposium on Tribo-Fatigue ISTF 2010, Part 2, Minsk, BGU, 399-404

18. Piskozub L.G., 2014, Longitudinal shear by concentrated force of bi-material with taking into account friction in the interfacial crack (in Ukrainian), Physic-Mathematical Modeling and Informational Technologies, 20, 160-172

19. Piskozub J.Z., Sulim G.T., 2008, Thermoelastic equilibrium of piecewise homogeneous solids with thin inclusions, Journal of Engineering Mathematics. Special Issue Thermomechanics, 61, $315-337$

20. Piskozub L.G., Sulym H.T., Pasternak Ya.M., 2014, Influence of friction on hysteresis under cycling loading of solid with interface crack by the longitudinal shear (in Ukrainian), Applied Problems of Mechanics and Mathematics, 12, 184-191

21. Sulym H.T., 2007, Bases of Mathematical Theory of Thermo-Elastic Equilibrium of Solids Containing Thin Inclusions (in Ukrainian), L'viv, Research and Publishing center of NTSh

22. Sulym G.T., Piskozub J.Z., 2004, Contact conditions (a survey) (in Ukrainian), Mat. Metody $i$ Fiz.-Meh. Polya (Journal of Mathematical Science), 47, 3, 110-125

23. Weertman J., Lin I.-H., Thomson R., 1983, Double slip plane crack model, Acta Metallurgica, 31, 4, 473-482 